\title{
Sexually Transmitted Infections: Risk-Factors among Married Female Patients' in Assiut, Egypt
}

\section{Essam A. El-Moselhy, ${ }^{1, *}$ MD,Lotfi F. Issa ${ }^{2}$ MD, Mohamed Abdelsattar ${ }^{3}$ MD,Manal G. Abdelati ${ }^{3}$ MD, Ramadan M. Eldhshan ${ }^{4}$ MD, Abdullah A. Mekky ${ }^{5}$ MD,Hesham H. Amin ${ }^{6}$ MD,Mohamed A. Bashir ${ }^{6}$ MD,Bahaa M. Badr $^{7}$ MD,and Ahmed S. Al Saadawy ${ }^{8}$ MD.}

*Corresponding Author:

Essam A. El-Moselhy

dr_elmoselhy@yahoo.com

Received for publication August8,2020; Accepted November 14, 2020; Published onlineNovember15, 2020.

Copyright 2020 The Authors published by Al-Azhar University, Faculty of Medicine, Cairo, Egypt. All rights reserved. This an openaccess article distributed under the legal terms, where it is permissible to download and share the work provided it is properly cited. The work cannot be changed in anyway or used commercially.

doi:10.21608/aimj.2020.38500.1295

${ }^{1}$ Department of Community Medicine, AssiutFaculty of Medicine, Al-Azhar University, Egypt

${ }^{2}$ Department of Community Medicine, CairoFaculty of Medicine, Al-Azhar University, Egypt

${ }^{3}$ Department of Obstetrics and Gynecology, AssiutFaculty of Medicine, Al-Azhar University, Egypt

${ }^{4}$ Department of Dermatology, Venereology \&Andrology, Damietta Faculty of Medicine, Al-Azhar University, Egypt

${ }^{5}$ Department of Psychiatry Faculty of Medicine, Al-Azhar University, Egypt

${ }^{6}$ Department of Clinical PathologyAssiutFaculty of Medicine, Al-Azhar University, Egypt

${ }^{7}$ Department of Microbiology and Immunology,AssiutFaculty of Medicine, Al-Azhar University, Egypt

${ }^{8}$ Department of Medical Parasitology,AssiutFaculty of Medicine, Al-Azhar University, Egypt

\section{ABSTRACT}

Background: Sexually transmitted infections (STIs) are major concern and public health problem worldwide. Risk-factors of STIs are numerous, their determination is basic for STIs prevention and control. Objective:To determine the commonest STIs and to define the socioeconomic, gynecological, reproductive, lifestyle, and behavioral risk-factors of the studied STIs female patients.

Patient and methods: The study was conducted on one-hundred STIs married female patients attending the Obstetrics\& Gynecology Clinics, Al-Azhar University Hospital, Assiut and one-hundred female as controls. A case-control study design was used. Vaginal, cervical, and urethral swabs and smears were taken from the cases and controls. Scrapes from suspicious lesions together with blood samples were taken for examinations.

Results:The commonest diagnosed STIs were candidiasis (38.0\%) and scabies (21.0\%). Significant risk-factors for acquiring STIs were the lowest positions of education, income and social-position (ORs $=2.02$, 2.42, 2.29; respectively). Urban- and shared-residence were significant risk-factors $\quad(\mathrm{ORs}=4.0, \quad 2.1 ; \quad$ respectively). Significant gynecologic/reproductive risk-factors were married at age $\geq 28$ years and $\geq 14$ years between menses and marriage (ORs $=2.15,2.17$; respectively). Pre-marital sexual activities, practicing with symptomatic partners, and had $\geq 2$ life-time sexual partners were significant risk-factors $(\mathrm{OR}=6.89$, 4.13, 4.75; respectively). Non-religiously committed, smoking, and substance-use/alcohol-intake were significant risk-factors (ORs=7.63, 14.1, 9.33; respectively). Not using protective measures, had previous STIs, partners with previous STIs, not advising partner to seek counseling, and counseling of non-healthcare were significant riskfactors (OR=3.73, 27.59, 11.16, 7.7, 24.41; respectively).

Conclusion:There are many preventable risk-factors for acquiring STIs; socioeconomic, demographic, gynecological, reproductive, sexual behavior, lifestyle, and healthcare behavior.

Keywords:STIs; Married females; Risk-factors; Egypt.

Disclosure: The authors have no financial interest to declare in relation to the content of this article. The Article Processing Charge was paid for by the authors.

Authorship: All authors have a substantial contribution to the article.

Sexually transmitted infections (STIs) are major concern and public health problem for developing and developed countries ${ }^{1,2}$. STIs are common infections; incidence continue to raise ${ }^{3,4}$. Annually, 500 million newly discovered cases of the commonest curable STIs occurring globally ${ }^{5}$. Millions of viral STIs occurs worldwide ${ }^{6}$. Women are fragile to the effects of STIs ${ }^{7}$.

In Egypt, STIs are main health, social, and economic load. Diagnosis is basically etiologic and healthcare based on clinical cure. STIs prevalence among rural women is high8; $3.0 \%$ of females using familyplanning unites, $4.0 \%$ of females using ante-natal care unites, and $5.4 \%$ of substance-use had 
minimally one STI. ${ }^{9}$ STIs prevalence in Egypt is up to $3.0 \%$ among $15-49$ years married females. ${ }^{10}$

STIs are major cause of acute ailment, disability, and death. ${ }^{6}$ Healthcare for STIs sequelae counts for great costs for cervical cancer treatment, infertility investigations, etc. ${ }^{11}$. Load in developing regions is expensive; $17.0 \%$ of economy of mal-health ${ }^{12}$. STIs were defined as risk-factors for human immunodeficiency virus (HIV) ${ }^{13}$.

Epidemiology of STIs is deficient in developing countries to many reasons e.g., socio-cultural (stigma), poverty, and inefficient diagnostic facilities ${ }^{14}$. Many STIs are asymptomatic; reported cases, even in developed regions, represent $50.0 \%$ $80.0 \%$ of actual numbers ${ }^{15}$.

STIs have common risk-factors and particular populations are liable to specified STIs ${ }^{6}$. Female gender is a risk-factor to many STIs $^{7}$. Lower socioeconomic-position is proven risk-factor for numerous STIs as Trichomonasvaginalis (TV $)^{6}$. Poverty, minimum access to healthcare, and homelessness/unstable residence may influence woman's sexual risk ${ }^{16}$.In Egypt; STIs types, spread, and risk-factors are rising as the results of socioculture, economic, sexual and healthcare behaviors risk-factors, and substance-use. These factors are influenced by Arabic conservative culture, male superiority, and female sexually conservative. Studies on these risk-factors are scarce ${ }^{17}$.

This study aims to define the commonest prevalent STIs among married female patients attending Gynecology-Obstetrics Clinics (GOCs), Al-Azhar University Hospital, Assiut, Egypt and to determine STIs socioeconomic, gynecological, reproductive, sexual and healthcare behaviors, and lifestyle riskfactors.

\section{PATIENTS AND METHODS}

I. Study type: Case-control, clinic-based.

II. Study setting and time: Was conducted at GOCs, Al-Azhar University Hospital, Assiut, from January 2019-2020.

III. Ethical considerations: Study protocol was approved by Research Ethics Committee of Assiut Faculty of Medicine, Al-Azhar University. Aims and procedures were cleared to the participants, confidentiality was assured, and informed consents were obtained.

\section{Study participants:}

1. The patients: Fisher's equation ${ }^{18}$ was used to define patients' sample size. STIs prevalence in Egypt was estimated at $5.4 \%{ }^{9}$. The calculated number was 79; increased to 100 to guard against patients with incomplete data.

1.1. Inclusion criteria: Married (currently/previously), aged >18years, practiced sexual activities (any form during the last 3months), with symptoms of STIs and confirmed by clinical and/or laboratory diagnosis.
2. The controls: One-hundred married female, STIs free, age-matched, and attendants' same clinics.

V. Study tools and methods: Patients and controls were subjected to:

1. Interviewing form: Specially designed, comprehensive form was used. Socioeconomic level was determined according to El-Gilanyet al. ${ }^{19}$.

2. Psychiatric assessment: Participants were surveyed by standardized, structured questionnaire according to American Psychiatric Association ${ }^{20}$ to eliminate participants with psychiatric disorders except substance-use.

3. Clinical examinations: Standardized examinations of the body. Attention was paid to lesions of urethra, vulva, vagina, cervix, vaginal discharge characteristics, uterine and adnexal tenderness, groin, and anal region. Controls with STIs were inserted to the patients' group.

4. Clinical and/or laboratory diagnosis:

4.1. Genital warts (GW) and Molluscumcontagiousm (MC): Were diagnosed clinically.

4.2. Genital herpes (GH): Was diagnosed clinically and laboratory confirmed by detection of specific antibodies for Herpes simplex virus (HSV) type I \&II using HerpeSelect ELISA-kit (USA, kit-lot EL0910G-5 for HSV/I and kit-lot EL0920G-5 for HSV/II). Absorbance of each sample-well was read at $450 \mathrm{~nm}$ on ELISA-reader (stat-fax2100, USA)

4.3. Scabies and Phthirus pubis (PP): Were diagnosed clinically; positive-history of infested husband was a precondition to determine sexual transmission.

4.3.1. Scabies was confirmed by demonstration of mites, eggs or scybala in scrapes from infested papules; scarped materials with 1-2drops of mineraloil was microscopically examined ${ }^{21}$.

4.3.2. PP; pubic hair was examined neatly by handlens to inspect infestation with adult-lice or viablenits ${ }^{21}$.

4.4. HBs-Ag and HIV-Abs serologic tests were done to diagnose HBV and HIV infections, respectively using Diagnostic Bioprobes ELISA-kit (Italy) for HBV (HBs-Ag kit-lot C4T5/1) and HIV (HIV-Ab kit-lot C7E7T6/11). Absorbance of each sample-well wasread at 450nm on ELISA-reader (stat-fax2100, USA). Husbands with HBV and/or HIV infection and without other parenteral causes of transmission were preconditions to determine sexual transmission.

4.5. Urethral, vaginal, and cervical swabs were obtained from all patients. Smears and/or swabs from suspected lesions plus blood samples were taken from patients for different microbiologic and serologic examinations ${ }^{22}$.

4.6. Patients were microscopically surveyed for Candida albicans, TV, N. gonorrhea, and bacterial vaginosis $(\mathrm{BV})$. 
For patients with cervical infections, microscopic examination was done for Gram-stained smears of cervical discharge. Cervical discharge swabs were immediately plated on chocolate-agar and incubated at $35^{\circ} \mathrm{C}$ in $5.0 \% \mathrm{CO}_{2}$ for gonorrhea and on bloodagar at $37^{\circ} \mathrm{C}$ for other bacterial pathogens. Growth on chocolate-agar was tested for Gram-negative diplococci and full identified by oxidase-test (+ve) and sugar fermentation-test (ferment only glucose). Growth on blood-agar plates was identified by colonial morphology, Gram-stain, coagulase- and catalase-tests for Gram-positive cocci. Ability to grow on bile salt-agar and sugar fermentation-tests were done for Gram-negative bacilli. Staphylococci are catalase-positive, while streptococci are negative. Coagulase-negative Staphylococcus saprophyticus was distinguished from S. epidermidis by its resistance to novobiocin. Group B streptococci infection was identified by Gram-stain, culture on blood-agar giving beta hemolysis and by the CAMP reaction; arrow head-shaped area of enhanced hemolysis when Str. agalactiae is inoculated perpendicular to a streak of Staph. aureus grown on blood-agar. Sera obtained from patients negative for microscopic and culture results were tested for gonococcal-Ags using LifeSpanBioSciences ELISAkit (USA) for N. gonorrhea (N. gonorrhea-Ab kit-lot LS-C73232). Absorbance of each sample-well was read at $450 \mathrm{~nm}$ on ELISA-reader (stat-fax2100, USA).

For candidiasis, direct Gram-stained smear of vaginal secretions was tested for Candida albicans buddingyeasts and further identified by culture on Sabaroud's agar and germ-tube test.

TV was detected by wet mount and/or by culture usingInPouchTV ${ }^{23}$.

Non-gonococcal cervicitis (NGCC) cases were tested microscopically for Chlamydia trachomatis (CT) infection by vaginal wet-mount and detection of chlamydia IgM using Diagnostic Bioprobes ELISAkit (Italy) for CT (kit-lot CT055M). Absorbance of each sample-well was read at $450 \mathrm{~nm}$ on ELISAreader (stat-fax2100, USA).

$\mathrm{BV}$ was detected by increasing vaginal $\mathrm{pH}$, positive Whiff-test (adding small amount of $\mathrm{KOH}$ to microscopic slide containing vaginal discharge; characteristic fishy-odor occurs), presence of cluecells, and quantitativemorphology of Gram-stained slides $^{24}$.

\section{Statistical analysis:}

Data were statistically analyzed using the Statistical Package for Social Science, version20. Qualitative data was described as frequency and percentage and analyzed using Chi-square $\left(\chi^{2}\right)$ or Fisher-exact (FE) test as appropriate. Odds ratio (OR) was used to define the risk. The significance level for $\chi^{2}$ and FE was accepted at p-value $<0.05$. For OR, 95\%- confidence interval (CI) or 95\%-exact confidence limits (ECL) was used as appropriate.

\section{RESULTS}

The commonest prevalent STIs were candidiasis (38.0\%), scabies (21.0\%), GW (14.0\%), and trichomoniasis (14.0\%) (table1).

\begin{tabular}{|l|c|c|}
\hline \multirow{2}{*}{ Types of STIs } & \multicolumn{2}{|c|}{$\begin{array}{c}\text { STIs female patients } \\
\text { (n=100) }\end{array}$} \\
\cline { 2 - 3 } & Number & Percent \\
\hline Candidiasis & 38 & 38.0 \\
\hline Scabies & 21 & 21.0 \\
\hline Genital warts & 14 & 14.0 \\
\hline Trichomoniasis & 14 & 14.0 \\
\hline Non-gonococcal cervicitis: & 12 & 12.0 \\
- Gram +vecocci & 3 & 3.0 \\
$\quad$ - Gram -ve bacilli & 4 & 4.0 \\
- Chlamydia trachomatis & 5 & 5.0 \\
\hline Bacterial vaginosis & 11 & 11.0 \\
\hline Molluscumcontagiosum & 9 & 9.0 \\
\hline Genital herpes & 8 & 8.0 \\
\hline Mixed vaginitis (fungal \&protozoal) & 8 & 8.0 \\
\hline Gonococcal cervicitis & 7 & 7.0 \\
\hline Hepatitis B virus infection & 6 & 6.0 \\
\hline Phthirus pubis & 5 & 5.0 \\
\hline Acquired immune-deficiency & 1 & 1.0 \\
syndrome (AIDS) & 18 & 18.0 \\
\hline Presence of two STIs & 5 & 5.0 \\
\hline Presence of $\geq 3$ STIs & 153 & --- \\
\hline Total number of diagnosis & & \\
\hline
\end{tabular}

Table 1: Frequency distribution of sexually transmitted infections (STIs) among sample of married Egyptian female patients

Lowest positions of education, income, and social level (ORs=2.02, 2.42, 2.29; respectively) were significant socioeconomic risk-factors. House-wife, ex-married, and urban residence were significant risk-factors (ORs $=2.42,2.12,4.0$; respectively) (table2).

Females married at age $\geq 28$ years, $\geq 14$ years between menses and marriage, and using of contraceptives were significant gynecologic/reproductive riskfactors (ORs=2.15, 2.17, 2.06; respectively) (table3).

Pre-marital sexual activities, had $\geq 2$ life-time sexual partners, and interchange sex for money/gifts were significant risk-factors (ORs=6.89, 4.75, 18.86; respectively) (table4).

Non-religiously committed, smoking, and substanceuse and/or alcohol-intake were significant riskfactors (ORs=7.63, 14.1, 9.33; respectively) (table5).

Patients had previous STIs, partners had previous STIs, and counseling of non-healthcare were significant risk-factors (ORs=27.59, 11.16, 24.41; respectively). Using clothes and sex avoidance as protection were significantly different between cases and controls ( $\mathrm{P}=0.008,0.004$, respectively) (table6). 


\begin{tabular}{|c|c|c|c|c|c|}
\hline \multirow{2}{*}{ Socioeconomic variables } & \multicolumn{2}{|c|}{$\begin{array}{c}\text { Cases } \\
(\mathrm{n}=100)\end{array}$} & \multicolumn{2}{|c|}{$\begin{array}{l}\text { Controls } \\
(\mathrm{n}=100)\end{array}$} & \multirow{2}{*}{$\begin{array}{c}\text { OR(95\% CI) } \\
\text { OR(95\% ECL) }\end{array}$} \\
\hline & No. & $\%$ & No. & $\%$ & \\
\hline \multicolumn{6}{|l|}{ Educational status: } \\
\hline Illiterate/read and write & 76 & 76.0 & 61 & 61.0 & $2.02(1.05-3.9)^{*}$ \\
\hline Elementary & 21 & 21.0 & 31 & 31.0 & $0.59(0.3-1.18)^{*}$ \\
\hline Secondary \& university & 3 & 3.0 & 8 & 8.0 & $0.36(0.06-1.55)^{* *}$ \\
\hline \multicolumn{6}{|l|}{ Occupation: } \\
\hline House-wife & 74 & 74.0 & 54 & 54.0 & $2.42(1.28-4.6)^{*}$ \\
\hline Working & 26 & 26.0 & 46 & 46.0 & $0.41(0.22-0.78)^{*}$ \\
\hline \multicolumn{6}{|l|}{ Income: } \\
\hline Not enough & 74 & 74.0 & 54 & 54.0 & $2.42(1.28-4.6)^{*}$ \\
\hline Enough & 25 & 25.0 & 40 & 40.0 & $0.5(0.26-0.95)^{*}$ \\
\hline Enough and save & 1 & 1.0 & 6 & 6.0 & $0.16(0.0-1.35)^{* *}$ \\
\hline \multicolumn{6}{|l|}{ Social level: } \\
\hline Low & 76 & 76.0 & 58 & 58.0 & $2.29(1.2-4.41)^{*}$ \\
\hline Middle & 23 & 23.0 & 36 & 36.0 & $0.53(0.27-1.03)^{*}$ \\
\hline High & 1 & 1.0 & 6 & 6.0 & $0.16(0.0-1.35)^{* *}$ \\
\hline \multicolumn{6}{|l|}{ Age (year): } \\
\hline $34-49$ & 57 & 57.0 & 59 & 59.0 & $0.92(0.51-1.68)^{*}$ \\
\hline \multicolumn{6}{|l|}{ Marital state: } \\
\hline Currently married & 56 & 56.0 & 73 & 73.0 & $0.24(0.11-0.53)^{*}$ \\
\hline Ex-married & 44 & 44.0 & 27 & 27.0 & $2.12(1.13-4.02)^{*}$ \\
\hline \multicolumn{6}{|l|}{ Place of residence: } \\
\hline Urban & 78 & 78.0 & 47 & 47.0 & $4.0(2.07-7.76)^{*}$ \\
\hline Rural & 22 & 22.0 & 53 & 53.0 & $0.25(0.13-0.48)^{*}$ \\
\hline \multicolumn{6}{|l|}{ Shared residence: } \\
\hline Yes & 33 & 33.0 & 19 & 19.0 & $2.1(1.04-4.24)^{*}$ \\
\hline No & 67 & 67.0 & 81 & 81.0 & $0.48(0.24-0.96)^{*}$ \\
\hline \multicolumn{6}{|l|}{ Husband travelling abroad: } \\
\hline No & 91 & 91.0 & 96 & 96.0 & $0.42(0.09-1.58)^{* *}$ \\
\hline \multicolumn{6}{|l|}{$\begin{array}{l}\text { Family troubles } \\
\text { husbands' violence: }\end{array}$} \\
\hline Present & 11 & 11.0 & 4 & 4.0 & $2.97(0.84-13.17)^{* *}$ \\
\hline Absent & 89 & 89.0 & 96 & 96.0 & $0.34(0.08-1.20)^{* *}$ \\
\hline
\end{tabular}

*Odds ratio, confidence interval

**Exact confidence limits

Table 2: Socioeconomic and demographic risk-factors of sexually transmitted infections among sample of married Egyptian female patients 


\begin{tabular}{|l|c|c|c|c|c|}
\hline \multirow{2}{*}{$\begin{array}{c}\text { Gynecological and } \\
\text { reproductive risk-factors }\end{array}$} & \multicolumn{2}{|c|}{$\begin{array}{c}\text { Cases } \\
(\mathrm{n}=100)\end{array}$} & \multicolumn{2}{c|}{$\begin{array}{c}\text { Controls } \\
(\mathrm{n}=100)\end{array}$} & \multirow{2}{*}{ OR(95\% CI) ${ }^{*}$} \\
\cline { 2 - 5 } & No. & $\%$ & No. & $\%$ & \\
\hline $\begin{array}{l}\text { Age at menses (year): } \\
\leq 14\end{array}$ & 59 & 59.0 & 43 & 43.0 & $1.91(1.05-3.48)$ \\
$>14$ & 41 & 41.0 & 57 & 57.0 & $0.52(0.29-0.96)$ \\
\hline Age at marriage (year): & 9 & 9.0 & 21 & 21.0 & $0.37(0.15-0.92)$ \\
$18-22$ & 48 & 48.0 & 53 & 53.0 & $0.82(0.45-1.48)$ \\
$23-27$ & 43 & 43.0 & 26 & 26.0 & $2.15(1.13-4.08)$ \\
$\geq 28$ & & & & & \\
\hline Time elapsed from menses to & & & & & \\
marriage (year): & 53 & 53.0 & 71 & 71.0 & $0.46(0.25-0.86)$ \\
$\leq 4$ & 47 & 47.0 & 29 & 29.0 & $2.17(1.16-4.06)$ \\
\hline 14 & & & & & \\
\hline Life-time pregnancies: & 14 & 14.0 & 11 & 11.0 & $1.32(0.53-3.32)$ \\
0 & 33 & 33.0 & 49 & 49.0 & $0.51(0.28-0.94)$ \\
$1-2$ & 53 & 53.0 & 40 & 40.0 & $1.69(0.93-3.08)$ \\
$\geq 3$ & & & & & \\
\hline Currently pregnant: & 86 & 86.0 & 83 & 83.0 & $1.26(0.55-0.92)$ \\
No & 14 & 14.0 & 17 & 17.0 & $0.79(0.34-1.83)$ \\
Yes & & & & & \\
\hline Current use of contraceptives: & 58 & 58.0 & 74 & 74.0 & $0.49(0.26-0.92)$ \\
No & 42 & 42.0 & 26 & 26.0 & $2.06(1.09-3.92)$ \\
\hline Yes & & & & &
\end{tabular}

*Odds ratio, confidence interval

Table 3: Gynecological and reproductive risk-factors of sexually transmitted infections among sample of married Egyptian female patients.

\begin{tabular}{|c|c|c|c|c|c|}
\hline \multirow{2}{*}{$\begin{array}{l}\text { Sexual behavior risk- } \\
\text { factors }\end{array}$} & \multicolumn{2}{|c|}{$\begin{array}{c}\text { Cases } \\
(\mathrm{n}=100)\end{array}$} & \multicolumn{2}{|c|}{$\begin{array}{l}\text { Controls } \\
(\mathrm{n}=100)\end{array}$} & \multirow{2}{*}{$\begin{array}{c}\text { OR(95\% CI) } \\
\text { OR(95\% } \\
\text { ECL) }{ }^{* *}\end{array}$} \\
\hline & No. & $\%$ & No. & $\%$ & \\
\hline $\begin{array}{ll}\text { Pre-marital sexual } \\
\text { activities: } \\
\text { No } \\
\text { Yes }\end{array}$ & $\begin{array}{l}54 \\
46\end{array}$ & $\begin{array}{l}54.0 \\
46.0\end{array}$ & $\begin{array}{l}89 \\
11\end{array}$ & $\begin{array}{l}89.0 \\
11.0\end{array}$ & $\begin{array}{l}0.15(0.06- \\
0.32)^{*} \\
6.89(3.12- \\
15.51)^{*}\end{array}$ \\
\hline $\begin{array}{l}\text { Sexual activities with } \\
\text { symptomatic partner: } \\
\text { No } \\
\text { Yes } \\
\end{array}$ & $\begin{array}{l}71 \\
29 \\
\end{array}$ & $\begin{array}{l}71.0 \\
29.0 \\
\end{array}$ & $\begin{array}{c}91 \\
9 \\
\end{array}$ & $\begin{array}{c}91.0 \\
9.0 \\
\end{array}$ & $\begin{array}{c}0.24(0.1-0.58)^{*} \\
4.13(1.73- \\
10.09)^{*} \\
\end{array}$ \\
\hline $\begin{array}{l}\text { Type of sexual activitis: } \\
\text { Vaginal } \\
\text { Non-vaginal (inter- } \\
\text { femoris, oral, etc.) }\end{array}$ & $\begin{array}{l}89 \\
11\end{array}$ & $\begin{array}{l}89.0 \\
11.0\end{array}$ & $\begin{array}{c}96 \\
4\end{array}$ & $\begin{array}{c}96.0 \\
4.0\end{array}$ & $\begin{array}{c}0.34(0.08-1.2)^{* *} \\
2.97(0.84- \\
13.17)^{* *} \\
\end{array}$ \\
\hline 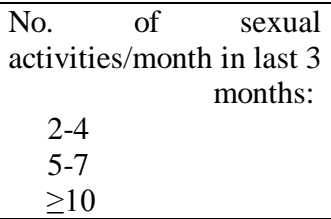 & $\begin{array}{l}27 \\
32 \\
41\end{array}$ & $\begin{array}{l}27.0 \\
32.0 \\
41.0\end{array}$ & $\begin{array}{l}16 \\
61 \\
23\end{array}$ & $\begin{array}{l}16.0 \\
61.0 \\
23.0\end{array}$ & $\begin{array}{c}1.94(0.92- \\
4.12)^{*} \\
0.3(0.16-0.56)^{*} \\
2.33(1.21-4.5)^{*}\end{array}$ \\
\hline $\begin{array}{l}\text { No. of life-time sexual } \\
\text { p a } \mathrm{r} \text { e } \mathrm{n} \text { e } \mathrm{r} \text { s: } \\
1 \\
\geq 2\end{array}$ & $\begin{array}{l}63 \\
37\end{array}$ & $\begin{array}{l}63.0 \\
37.0\end{array}$ & $\begin{array}{l}89 \\
11\end{array}$ & $\begin{array}{l}89.0 \\
11.0\end{array}$ & $\begin{array}{c}0.21(0.09- \\
0.47)^{*} \\
4.75(2.14- \\
10.77)^{*}\end{array}$ \\
\hline $\begin{array}{l}\text { Interchange sex for } \\
\text { benefits e.g. gifts and/or } \\
\text { money: } \\
\text { No } \\
\text { Yes }\end{array}$ & $\begin{array}{l}84 \\
16\end{array}$ & $\begin{array}{l}84.0 \\
16.0\end{array}$ & $\begin{array}{c}99 \\
1\end{array}$ & $\begin{array}{c}99.0 \\
1.0\end{array}$ & $\begin{array}{c}0.05(0.0-0.36)^{* *} \\
18.86(2.79- \\
798.56)^{* *}\end{array}$ \\
\hline
\end{tabular}

Table 4: Sexual behavior risk-factors of sexually transmitted infections among sample of married Egyptian female patients 


\begin{tabular}{|c|c|c|c|c|c|}
\hline \multirow{2}{*}{ Lifestyle risk-factors } & \multicolumn{2}{|c|}{$\begin{array}{l}\text { Cases } \\
(\mathrm{n}=100)\end{array}$} & \multicolumn{2}{|c|}{ Controls $(n=100)$} & \multirow{2}{*}{$\begin{array}{c}\text { OR(95\% CI })^{*} \\
\text { OR(95\% ECL) }\end{array}$} \\
\hline & No. & $\%$ & No. & $\%$ & \\
\hline $\begin{array}{l}\text { Religiously committed: } \\
\text { Yes } \\
\text { Fair } \\
\text { No }\end{array}$ & $\begin{array}{l}27 \\
22 \\
51\end{array}$ & $\begin{array}{l}27.0 \\
22.0 \\
51.0\end{array}$ & $\begin{array}{l}57 \\
31 \\
12\end{array}$ & $\begin{array}{l}57.0 \\
31.0 \\
12.0\end{array}$ & $\begin{array}{l}0.28(0.15-0.53)^{*} \\
0.63(0.32-1.24)^{*} \\
7.63(3.53-16.77)^{*}\end{array}$ \\
\hline $\begin{array}{l}\text { Cigarettes and/or sheesha smoking: } \\
\text { Yes } \\
\text { Sometimes } \\
\text { No }\end{array}$ & $\begin{array}{l}37 \\
49 \\
14 \\
\end{array}$ & $\begin{array}{l}37.0 \\
49.0 \\
14.0\end{array}$ & $\begin{array}{c}4 \\
23 \\
73 \\
\end{array}$ & $\begin{array}{c}4.0 \\
23.0 \\
73.0\end{array}$ & $\begin{array}{c}14.1(4.67-56.41)^{* *} \\
3.22(1.68-6.2)^{*} \\
0.06(0.03-0.13)^{*}\end{array}$ \\
\hline $\begin{array}{l}\text { Substance-use and/or alcohol-intake: } \\
\text { Yes } \\
\text { No }\end{array}$ & $\begin{array}{l}48 \\
52\end{array}$ & $\begin{array}{l}48.0 \\
52.0\end{array}$ & $\begin{array}{c}9 \\
91\end{array}$ & $\begin{array}{c}9.0 \\
91.0\end{array}$ & $\begin{array}{c}9.33(4.201-22.31)^{*} \\
0.11(0.04-0.25)^{*}\end{array}$ \\
\hline $\begin{array}{l}\text { Engaging in criminal activities: } \\
\text { Yes } \\
\text { No }\end{array}$ & $\begin{array}{c}8 \\
92\end{array}$ & $\begin{array}{c}8.0 \\
92.0\end{array}$ & $\begin{array}{c}2 \\
98\end{array}$ & $\begin{array}{c}2.0 \\
98.0\end{array}$ & $\begin{array}{c}4.26(0.82-41.95)^{* *} \\
0.23(0.02-1.23)^{* *}\end{array}$ \\
\hline
\end{tabular}

*Odds ratio, confidence interval $\quad * *$ Exact confidence limits

Table 5: Lifestyle risk-factors of sexually transmitted infections among sample of married Egyptian female patients

\begin{tabular}{|c|c|c|c|c|c|}
\hline \multirow[t]{2}{*}{ Healthcare behaviors risk-factors } & \multicolumn{2}{|c|}{$\begin{array}{l}\text { Cases } \\
(\mathrm{n}=100)\end{array}$} & \multicolumn{2}{|c|}{$\begin{array}{l}\text { Controls } \\
(\mathrm{n}=100)\end{array}$} & \multirow{2}{*}{$\begin{array}{c}\mathrm{OR}(95 \% \mathrm{CI})^{*} \\
\mathrm{OR}(95 \% \mathrm{ECL})^{* *} \\
\chi^{2^{\#}-\mathrm{FE}^{\# \#}} \\
\end{array}$} \\
\hline & No. & $\%$ & No. & $\%$ & \\
\hline $\begin{array}{l}\text { Using preventive/protective measure if partner suspect } \\
\text { STI: } \\
\text { No } \\
\text { Sometimes } \\
\text { Yes }\end{array}$ & $\begin{array}{l}58 \\
36 \\
6\end{array}$ & $\begin{array}{c}58.0 \\
36.0 \\
6.0\end{array}$ & $\begin{array}{l}27 \\
42 \\
31\end{array}$ & $\begin{array}{l}27.0 \\
42.0 \\
31.0\end{array}$ & $\begin{array}{l}3.73(1.98-7.08)^{*} \\
0.78(0.42-1.43)^{*} \\
0.14(0.05-0.37)^{* *}\end{array}$ \\
\hline $\begin{array}{l}\text { The most common used preventive/protective measures: } \\
\text { Segregation by clothes } \\
\text { Sex avoidance } \\
\text { Others e.g. condom }\end{array}$ & $\begin{array}{l}79 \\
19 \\
2\end{array}$ & $\begin{array}{c}79.0 \\
19.0 \\
2.0\end{array}$ & $\begin{array}{c}61 \\
38 \\
1\end{array}$ & $\begin{array}{c}61.0 \\
38.0 \\
1.0\end{array}$ & $\begin{array}{c}\chi^{2^{\#}}=6.88, P=0.008 \\
\chi^{2^{\#}}=7.95, P=0.004 \\
F E^{\# \#}, P=1\end{array}$ \\
\hline $\begin{array}{l}\text { Knowing symptoms of STIs among males and females: } \\
\text { No } \\
\text { Yes }\end{array}$ & $\begin{array}{l}64 \\
36 \\
\end{array}$ & $\begin{array}{l}64.0 \\
36.0 \\
\end{array}$ & $\begin{array}{l}53 \\
47 \\
\end{array}$ & $\begin{array}{l}53.0 \\
47.0 \\
\end{array}$ & $\chi^{2^{\#}}=2.06, \mathrm{P}=0.151$ \\
\hline $\begin{array}{l}\text { You previously infected with STIs: } \\
\text { Yes } \\
\text { No }\end{array}$ & $\begin{array}{l}79 \\
21\end{array}$ & $\begin{array}{l}79.0 \\
21.0\end{array}$ & $\begin{array}{l}12 \\
88\end{array}$ & $\begin{array}{l}12.0 \\
88.0\end{array}$ & $\begin{array}{c}27.59(12.01-64.82)^{*} \\
0.4(0.02-0.08)^{*}\end{array}$ \\
\hline $\begin{array}{l}\text { Partner previously infected with STIs: } \\
\text { Yes } \\
\text { No }\end{array}$ & $\begin{array}{l}68 \\
32 \\
\end{array}$ & $\begin{array}{l}68.0 \\
32.0 \\
\end{array}$ & $\begin{array}{l}16 \\
84 \\
\end{array}$ & $\begin{array}{l}16.0 \\
84.0\end{array}$ & $\begin{array}{c}11.16(5.38-23.43)^{*} \\
0.09(0.04-0.19)^{*}\end{array}$ \\
\hline $\begin{array}{l}\text { Advice partner to seek counseling: } \\
\text { Yes } \\
\text { No }\end{array}$ & $\begin{array}{l}36 \\
64 \\
\end{array}$ & $\begin{array}{l}36.0 \\
64.0 \\
\end{array}$ & $\begin{array}{c}\mathrm{n}=16 \\
13 \\
3\end{array}$ & $\begin{array}{l}71.0 \\
29.0 \\
\end{array}$ & $\begin{array}{l}0.13(0.02-0.52)^{* *} \\
7.7(1.91-44.13)^{* *}\end{array}$ \\
\hline $\begin{array}{l}\text { Early counseling to manage suspected STIs: } \\
\text { Yes } \\
\text { No }\end{array}$ & $\begin{array}{l}74 \\
26\end{array}$ & $\begin{array}{l}74.0 \\
26.0\end{array}$ & $\begin{array}{c}\mathrm{n}=12 \\
11 \\
1\end{array}$ & $\begin{array}{l}89.0 \\
11.0\end{array}$ & $\begin{array}{l}0.26(0.01-1.96)^{* *} \\
3.86(0.51-172.65)^{* *}\end{array}$ \\
\hline $\begin{array}{l}\text { Source of first counseling for suspected STIs: } \\
\text { Healthcare professionals } \\
\text { Non-healthcare (partner, friends, relatives, etc.) }\end{array}$ & $\begin{array}{l}17 \\
83 \\
\end{array}$ & $\begin{array}{l}17.0 \\
83.0\end{array}$ & $\begin{array}{c}\mathrm{n}=12 \\
10 \\
2\end{array}$ & $\begin{array}{l}58.3 \\
41.7 \\
\end{array}$ & $\begin{array}{c}0.04(0.00-0.23)^{* *} \\
24.41(4.44-238.53)^{* *}\end{array}$ \\
\hline $\begin{array}{l}\text { History of compliance with treatment: } \\
\text { Yes } \\
\text { No }\end{array}$ & $\begin{array}{c}\mathrm{n}=79 \\
25 \\
54\end{array}$ & $\begin{array}{l}31.6 \\
68.4\end{array}$ & $\begin{array}{c}\mathrm{n}=3 \\
2 \\
1\end{array}$ & $\begin{array}{l}75.0 \\
25.0\end{array}$ & $\mathrm{FE}^{\# \#}, \mathrm{P}=0.251$ \\
\hline
\end{tabular}

Table 6: Healthcare behaviors' risk-factors of sexually transmitted infections (STIs) among sample of married Egyptian female patients 


\section{DISCUSSION}

Few data is obtainable on the extent of STIs in Egypt $^{25}$. In developing world, STIs present major health, social, and economic burden ${ }^{2}$.

This study reported $38.0 \%$ of patients had candidiasis. This is similar to El-Moselhy et al. ${ }^{26,27}$ and Geremew et al. ${ }^{(10)}$ figures; 35.6\%, 36.0\%, 32.5\%; respectively. Garget al. ${ }^{28}$ observed $19.0 \%$.

In the current study, it was found that $21.0 \%$ of the patients had scabies. This finding is double that of El-Moselhyet al. $^{26}(10.0 \%)$ and greatly higher than Sarkar et al. ${ }^{29}$ (1.8\%); who studied genital scabies, while whole-body scabieswas included in our study. Scabies is common among lowest socioeconomic population ${ }^{16}$, this is similar to our findings as the presence of risk-factors e.g. crowding, poor hygiene, and sexual muddle might increase its spread. Scabies is considered from patients' respect as non-STI and is presented in clinic with no stigma.

We showed $5.0 \%$ of our patients had PP. This figure is higher than El-Moselhy et al. ${ }^{26,27}$, Sarkar et al. ${ }^{29}$ figures'; $3.3 \%, 4.0 \%$, $0.9 \%$; respectively. PP is common among lowest socioeconomic populations; crowding, poor hygiene, and sexual muddle are dominated.

We claimed $14.0 \%$ of the patients had GW. Gewirtzmanet al. ${ }^{6}$ cleared human papilloma virus (HPV) infection is the commonest STI worldwide. Our figure is comparable to Boschet al. ${ }^{30}$; globally HPV prevalence, in normal cytology females is $\sim 10.0 \%$. Kenyon et al. ${ }^{31}$ stated HPV is $7.0 \%-13.6 \%$ in North Africa and Middle East. El-Moselhyet al. ${ }^{26}$ reported $13.0 \%$. Dunne et al. $^{32}$ reported $26.8 \%$ in USA.

We illustrated $7.0 \%$ of the patients had GCC. Geremewet al. ${ }^{14}$ and Pandey et al. ${ }^{33}$ found prevalence 20.8\%, 34.6\% in Ethiopia and Nepal; respectively. Korenrompet al. ${ }^{34}$ and El-Kettani et al. ${ }^{35}$ found $0.37 \%$, $3.8 \%$; respectively. These figures differences may be clarified; the small figure was reported among general population. High figures may be referred to differences regarding the studied populations and diagnostic methods. In Egypt, Ali et $\mathrm{al}^{36}$ and El-Moselhy et al. ${ }^{26,27}$ found N. gonorrhea was $26.0 \%, 3.3 \%, 6.0 \%$; respectively. The high figure represents GC urethritis and GCC. NGCC [Gram-positive cocci (3.0\%) and Gram-negative bacilli $(4.0 \%)$ ] was higher; $2.2 \%^{26}, 2.0 \%^{28}$, and $2.0 \%{ }^{27}, 4.0 \%{ }^{27}$ for each pathogen.

We showed CT infection was 5.0\%. In Egypt, CT prevalence was $1.3 \%-52.0 \%$ among different categories of patients ${ }^{37}$. Our figure is parallel to ElKettaniet al. ${ }^{35}$ (3.8\%) and Mosbah\&Nabiel ${ }^{38}$ (4.4\%) figures', higher than El-Moselhy et al. ${ }^{27}$ (3.0\%) and lower than Javanmard et al. ${ }^{39}(11.4 \%)$.

Bacterial vaginosis (BV) was detected in $11.0 \%$ of the patients had BV. Our figure is higher than ElMoselhy et al. ${ }^{26,27}$ (8.9\% and $8.0 \%$, respectively), double in the study of Pandey et al. ${ }^{33}(5.8 \%)$, and one-fourth that of Garg et al. ${ }^{28} ; 41.0 \%$.
MC was $9.0 \%$ among our patients. This is higher than El-Moselhy et al. ${ }^{26,27}$ and Sarkar et al. ${ }^{29}$ figures; $4.4 \%, 6.0 \%$, 3.7\%; respectively.

Trichomoniasis was $14.0 \%$ among our patients. Our figure is higher than Garget al. ${ }^{28} ; 4.0 \%$ and similar to Geremew et al. ${ }^{14} ; 14.2 \%$.

Eight-percent of the patients had genital herpes. Our figure is lower than that of Xuet al. ${ }^{40}(13.2 \%)$, Kenyon et al. ${ }^{31}$ (15.4\%), and Gottlieb et al. ${ }^{41}$ (52.0\%) figures'. It close to other studies $(6.7 \%, 7.0 \%$ and $8.4 \%$, respectively) $)^{(26,27,29)}$.

We claimed $4.0 \%$ of our patients had HBV infection. HBV sexual transmission is significant route for women's infection ${ }^{42}$. This figure is identical to our past figure; $4.0 \%{ }^{27}$.

We clarified $1.0 \%$ of our patients had acquired immune-deficiency syndrome (AIDS). Our figure is identical to Pandey'set al. ${ }^{33} ; 1.0 \%$ and higher than Kenyon et al. ${ }^{31}$ figures; (0.2\%-0.4\%).

The lowest educational level among our patients was a significant risk-factor. STIs are common among non-educated ${ }^{25,26}$. This accordant with El-Moselhyet al. $^{26}$; $(\mathrm{OR}=1.9)$ and Gottlieb et al. ${ }^{41}$ (OR=1.8). House-wife was significant risk-factor. This concordant with El-Moselhyet al. ${ }^{26}$. We cleared that no enough income was significant risk-factor; poverty might influence woman's sexual risk ${ }^{16}$. Our result was parallel to Kelly et al. ${ }^{16}$ and $\mathrm{Xu}$ et al. ${ }^{40}$; more HSV prevalence among under-poverty level population. Gottlieb et al. ${ }^{41}$ observed high HSV prevalence among minorities $(\mathrm{OR}=2.1)$. Conjointly, low social-position of our patients was significant risk-factor. This finding is concordant with Osman et al. ${ }^{25}$ and Xu et al. ${ }^{40}$. Gottlieb et al. ${ }^{41}$ didn't found this association. Social characters in developing communities are associated with STIs propagation ${ }^{6}$. These could be explained by poor economy in developing communities directed governments to expense little on healthcare and prevention programs; healthcare becomes low affordable to the patients. Urban residence, significant risk-factor, might be attributed to residence of slums. This leads to rise of illegal sex and STIs prevailing ${ }^{43}$. Shared- and unstable residence represented significant risk-factors; had impact on sexual mix-up, families can't spend conservative effect on individuals' socio-cultural behaviors e.g. sexuality and affects female's sexual risk $^{16}$. We showed ex-married patients, divorced, and widow, were significant risk-factor. Our result concordant with Aral et al. ${ }^{44}$ and Abdullah et al. ${ }^{45}$; they reported high risk of unmarried females. We could suppose married patient had her own husband. While, divorced and widow might involve in accidental relations. Family disturbances; husband's long travelling abroad and domestic violence were insignificant risk-factors; could be attributed to miss and/or hate of partner.

Age $\leq 14$ year at first menses and late age ( $\geq 28$ year) at marriage were a significant risk-factors for STIs acquisition. Globally, mean age at first menses has decreased $^{44}$. Moreover, >13years elapsed from menses to marriage was a significant risk-factor. These results could be explained; late marriage and 
much time elapsed till marriage might put females under stress. Socio-cultural changes have led to raise mean age at females' marriage. This elevation might led to many free sexual relations; many of them are pre-marital causal-sex ${ }^{44}$. In developing countries, like Egypt, with social and economic-position characterized by delay and economic inflation, overpopulation, slums, and retarded education and healthcare services fueling conditions leading to spread of STIs epidemic.

Contraceptive using was a significant risk-factor. This concordant with El-Moselhyet al. ${ }^{26}$. Intrauterine device (IUD) raise risk of genital tract infection by STIs; IUD facilitates biological infection mechanically.

Pre-marital sexual practice was significant riskfactor. Overtime rise in pre-marital relations has been certified. Alterations in sexual behavior have situated females at excess risk for STIs with the worldwide trend to early age at first coitus ${ }^{44}$. The increase in pre-marital sex was facilitated by the improvement of birth-control methods, advances in movement of the women's freedom, social situations and ideas enhanced delay marriage, women's entrance into the labor force, and elevated divorce rate. The major problem female's pose is inability to safeguard herself against STIs $^{44}$. Sexual activity with symptomatic partner was a significant risk-factor. This could be explained by low socioeconomicposition and poor sexual behavior. Scabies and PP didn't necessitate vaginal intercourse. There are some speculations about non-vaginal sexual activities; females might be used/accepted these practices as contraceptive methods. In Egypt, high risk sexual practices are un-agreeable ${ }^{25}$. We reported $89.0 \%, 11.0 \%$ of our patients have practiced vaginal and non-vaginal sex, respectively. Aral et al. ${ }^{44}$ cleared normal vaginal sexual practice is the commonest form; $\sim 80.0 \%$. El-Moselhyet $\mathrm{al}^{26}$ showed non-vaginal sex was more common among STIs patients; $\sim 20.0 \%$ of women experienced oral and/or anal sex. Frequency of sexual activities $\geq 10$ time/month was significant risk-factor. This could be explained; $56.0 \%$ of our patients were currently married; more ability to make sex. ElMoselhyet al. ${ }^{26}$ cleared $\sim 24.0 \%$ of STIs women had vaginal intercourse $\geq 12$ times/month. Life-time sexual partners' number $\geq 2$ was significant riskfactor. This consisted with Osman et al. ${ }^{25}$ and $\mathrm{Xu}$ et al. $^{40}$. Interchange sexual activities for benefits was a significant risk-factor. This accordant with ElMoselhyet al. ${ }^{26}$ and Abdullah et al. ${ }^{45}$; trade sex is prevalent in statuses recognized by poorness and social degradation, it represents great part in STIs epidemiology in most developing countries.

Religious non-commitment was significant riskfactor. This result concordant with El-Moselhyet al. ${ }^{26}$ and Abdullah et al. ${ }^{45}$. Commitment was significant protective-factor. In the Holy Quran, Allah strictly prohibited illegitimate relations and harlotry. Religious commitment has major role in sex avoidance till marriage. In Egypt, religion beliefs, cultural standards, and norms prohibit pre-marital and extra-marital sex. Smoking (cigarette/sheesha) and substance-use/alcohol-intake were significant risk-factor. These consistent with El-Moselhyet al. ${ }^{26}$ and Abdullah et al. ${ }^{45}$. Intravenous substance-use is un-agreeable high risk behavior; linked with increased STIs transmission ${ }^{25}$. Substanceuse/alcohol-intake is linked with risky sexual activities and behaviors; would raise risk of acquiring STIs ${ }^{26,45}$. Sharing in criminal/socially unaccepted activities history was insignificant risk-factor. Offenders are at risk of acquiring STIs ${ }^{26,46}$.

Partner (husband) not using preventive measures to guard against STIs was significant risk-factor. STIs are common among patients practicing unprotected $\operatorname{sex}^{10,25,26,44}$. The commonest methods for STIs prevention were the separation between partners with clothes and sex avoidance. The use of other protective measures as condom is scarce. This could be clarified; in developing areas, condom use is low, in Egypt it's uncommon method for prevention and contraception $^{10,25}$. There is males' unfavorable behavior opposed condom use. The shame of buying condoms might be another barrier. This result was consistent with Abdullah et al. ${ }^{45}$. Past-history of STIs among the patients was significant risk-factor. ElMoselhyet al. ${ }^{26}$, Gottlieb et al. ${ }^{41}$, and Aral et al. ${ }^{44}$ found that risk. Among husbands with past STIs infections, females might consider themselves immune or at minimum risk to catch STIs. In most developing countries STIs diagnosis and treatment for women are the greatest stigma ${ }^{17,25,26}$; these force women to conceal and abstain from seeking health appraisal and advice ${ }^{25}$. Divorced or widow females are more shame to seek healthcare for STIs. The shame about STIs might work as an obstacle to fast healthcare searching, related to case of confidentiality or searching to treat symptoms using self-care ${ }^{17,25}$. Women might carry quietly STIs symptoms without searching for healthcare. Were et al. ${ }^{47}$ confirmed value of detecting partners of STI patients; used to increase interventions and patients' treatment. Patients not advising their partners to request counseling was considered as risk-factor. This could be explained; STIs stigma, self-care, privacy, or occasional relations ${ }^{17,25,26}$. Early counseling and early diagnosis of husband with STIs could protect his wife. Non-professional healthcare personnel (partner, friends, relatives) counseling was significant risk-factor. Minimum access to effective healthcare may affects woman's sexual risk ${ }^{16}$. This might be explained by stigma, fear, and confidentiality ${ }^{17,25}$.

\section{CONCLUSION}

Candidiasis andscabies were the commonest STIs. STIs significant risk-factors were low socioeconomic-position, house-wife, urban residence, shared residence, late age at marriage, practice with symptomatic partner, use of contraceptives, pre-marital sexual activities, religiously non-committed, and patient and partner previous STIs. Define STIs risk-factors could help in their prevention and control. 


\section{REFERENCES}

1. Hughes $G$ and Field N. The epidemiology of sexually transmitted infections in the UK: impact of behavior, services and interventions. Future Microbiol. 2015; 10(1):35-51.

2. CDC (Centers for Disease Control and Prevention). Sexually Transmitted Disease Surveillance 2017. Atlanta: U.S. Department of Health and Human Services, 2018. Available at: https://www.cdc.gov/std/stats

3. Menezes CB, Frasson AP and Tasca T. Trichomoniasis: Are we giving the deserved attention to the most common non-viral sexually transmitted disease worldwide? Microb Cell. 2016; 3(9):404-18.

4. Chen X-S, Gong X-D, Liang G-J, et al. Epidemiologic trends of sexually transmitted diseases in China. Sex Trans Dis. 2000; 27(3): $138-42$.

5. WHO (World Health Organization).Global incidence and prevalence of selected curable sexually transmitted infections-2008. WHO, Geneva, Switzerland, 2012.

6. Gewirtzman A, Bobrick L, Conner K, et al. Epidemiology of sexually transmitted infections. In: Gross G and Tyring SK (Eds.), Sexually TtransmittedInfections and sexually Sexually Transmitted Diseases, Berlin Heidelberg: Springer-Verlag. 2011; pp. 13-34.

7. Jolly DH, Mueller MP, Chen M, et al. Concurrency and other sexual risk behaviors among black young adults in asSoutheastern city. AIDS Educ Prev. 2016; 28(1): 59-76.

8. WHO (World Health Organization). Report on the regional consultation to strengthen STD prevention and care strategies in the countries of the Eastern Mediterranean Region. WHO ROEM, 2001.

9. WHO (World Health Organization). Progress report on acquired immunodeficiency syndrome (AIDS) in the Eastern Mediterranean Region. 49 Session of the Regional Committee for the Eastern Mediterranean, 2002.

10. El-Zanaty F and Associates, Ministry of Health and Population [Egypt], and ICF International. Egypt Demographic and Health Survey 2014. Cairo, Egypt and Rockville, Maryland, USA: Ministry of Health and Population and ICF International, 2015. Available at: http://dhsprogram.com/pubs/pdf/fr302/fr302.pdf.

11. WHO (World Health Organization). Global strategy for the prevention and control of sexually transmitted infections- 2006-2015: Breaking the chain of transmission. WHO Press, Geneva; 2007.

12. Mayaud D and Mabey DCW. Approaches to the control of sexually transmitted infections in developing countries: Old problems and modern challenges. Sex Trans Infect. 2004; 80(3): 17482.

13. Marrazzo JM1, Dombrowski JC and Mayer KH. Sexually transmitted infections in the era of antiretroviral-based HIV prevention: Priorities for discovery research, implementation science, and community involvement. PLoS Med. 2018; 15(1): e1002485.

14. Geremew RA, Agizie BM, Bashaw AA, et al. Prevalence of selected sexually transmitted infection (STI) and associated factors among symptomatic patients attending Gondar Town Hospitals and Health Centers. Ethiop J Health Sci. 2017; 27(6): 589-600.

15. Da Rose CT and Schmitt CS. Global epidemiology of sexually transmitted disease. Asian J Androl. 2008; 10(1): 110-114.

16. Kelly J, Cohen J, Grimes B, et al. High rates of herpes simplex virus type 2 infection in homeless women: Informing public health strategies. $J$ Women's Health. 2016; 25(8): 840-5.

17. Bakhoum AY. HIV/AIDS, hepatitis and sexually-transmitted infection prevention among Egyptian substance users. Thesis submitted for the degree of Doctor of Philosophy, Norwich Medical School, East Anglia University, 2015.

18. Araoye MO. Sample size determination. In: Araoye MO (Ed.), Research Methodology with Statistics for Health and Social Sciences, 1st ed., Ilorin: NathadexPublishers, 2003; pp. 15-21.

19. El-Gilany A, El-Wehady A and El-Wasify M. Updating and validation of the socioeconomic status scale for health research in Egypt. EMHJ. 2012; 18: 962-8.

20. American Psychiatric Association. Diagnostic and statistical manual of mental disorders: DSM5. Washington, D.C: American Psychiatric Association, 2013.

21. Garcia LS and Procop GW. Diagnostic Medical Parasitology, Manualof Commercial Methods in Clinical Microbiology. In: Truant AL, Tang Y-W, Waites KB, et al. (Eds.), Manual of Commercial Methods in Clinical Microbiology, Chapter 15, International Edition, 2nded., John Wiley \& Sons, Inc., 2016; pp. 284308.

22. Riedel S and Hobden JA. Principles of diagnostic medical microbiology. In: Riedel S, Hobden JA, Miller S, et al. (Eds.); Jawetz, Melnick, Adelberg's Medical Microbiology, 28th ed., New York: McGraw-Hill Education, 2019; pp. 75785.

23. Sood S, Mohanty S, Kapil A, et al. InPouch TVTM culture for detection of Trichomonasvaginalis. Indian J Med Res. 2007; 125: 567-71.

24. Nugent RP, Krohn MA and Hillier S. Reliability of diagnosing bacterial vaginosisis improved by a standardized method of Gram-stain 
interpretation. J Clin Microbiol.1991; 29: 297301.

25. Osman M, Ragab AR, Girgis H, et al. Population situation analysis: Egypt 2016. ECPOR, UNFPA, NPC. Available at: https://egypt.unfpa.org`sites>default>files`pubpdf.

26. El-Moselhy EA, Khalifa HO, Abd-Allah KM, et al. Risk factors and impacts of sexually transmitted diseases among adult females in Cairo. Egypt J Hosp Med. 2005; 20: 112-30.

27. El-Moselhy EA, Hassan $\mathrm{H}$ and Amin $\mathrm{H}$. Demographic, socioeconomic characteristics and impacts of sexually transmitted diseases in adult Egyptians: A clinic based epidemiological study. Egypt J Med Sci. 2011; 32(2): 1375-95.

28. Garg S, Hana T, John L, et al. Reproductive morbidity in an Indian urban slum: Need for health action.Sex Trans Infect. 2002; 78:68-9.

29. Sarkar S, Shrimal A, Das J, et al. Pattern of sexually transmitted infections: A profile from a sexually transmitted infections clinic of a tertiary care hospital of eastern India. Ann Med Health Sci Res. 2013; 3: 206-9.

30. Bosch FX, Burchell AN, Schiffman M, et al. Epidemiology and natural history of human papillomavirus infections and type-specific implications in cervical neoplasia. Vaccine. 2008; 26(10): K1-16.

31. Kenyon C, Buyze $\mathrm{J}$ and Colebunders R. Classification of incidence and prevalence of certain sexually transmitted infections by world regions.Int J Infec Dis. 2014; 18: 73-4.

32. Dunne EF, Unger ER, Sterberg $M$, et al. Prevalence of HPV infection among females in the United States. JAMA. 2007; 297(8): 813-9.

33. Pandey S, Sharma N, Pokhrel N, et al. Pattern of sexually transmitted infections at Kohalpur Teaching Hospital. J Nepal Gunj Med College. 2015; 13(1): 25-27.

34. Korenromp EL, Mahiané G, Rowley J, et al. Estimating prevalence trends in adult gonorrhea and syphilis in low- and middle-income countries with the Spectrum-STI model: Results for Zimbabwe and Morocco from 1995 to 2016. Sex Transm Infect. 2017; 93: 599-606.

35. El-Kettani A, Mahiané G, Bennani A, et al. Trends in adult Chlamydia and Gonorrhea prevalence, incidence and urethral discharge case reporting in Morocco over 1995-2015: Estimates using the spectrum-sexually transmitted infection model. STDs. 2017; 44(9): 557-64.

36. Ali F, Aziz AA, Helmy MF, et al. Prevalence of certain sexually transmitted diseases in Egypt. $J$ Egypt Public Health Assoc. 1996; 71(5-6): 55375.
37. Smolak A, Chemaitelly H, Hermez JG, et al. Epidemiology of Chlamydia trachomatis in the Middle East and North Africa: A systematic review, meta-analysis, and meta-regression. Lancet Glob Health. 2019; 7: e1197-225.

38. Mosbah A and Nabiel Y. Helicobacter pylori, Chlamydiaepneumoniae and trachomatis as probable etiological agents of preeclampsia. $J$ Matern Fetal Neonatal Med. 2016; 29:1607-12.

39. Javanmard D, Behravan M, Ghannadkafi M, et al. Detection of Chlamydia trachomatis in Pap smear samples from south Khorasan province of Iran. Int J FertilSteril. 2018; 12: 31-36.

40. $\mathrm{Xu} \mathrm{F}$, Schillinger $\mathrm{M}$, Stermberg $\mathrm{R}$, et al. Seroprevalence and co-infection with herpes Herpes simplex virus type I and type II in the United States 1988-1999. J Infec Dis. 2002; 185(8): 1019-24.

41. Gottlieb SL, Douglas JM, Schmid DS, et al. Seroprevalence and correlates of herpes Herpes simplex virus type 2 infection in five sexually transmitted disease clinics. J Infec Dis. 2002; 185: 1381-9.

42. Lester LJ and Agarwal SK. Hepatitis viruses as sexually transmitted diseases. In: Gross G, Tyring SK (Eds.), Sexually Transmitted Infections and Sexually Transmitted Diseases, Berlin Heidelberg:,Springer-Verlag, 2011; pp. 553-85.

43. Amo J, Gonzalez C, Losana J, et al. Influence of age and geographical origin in the prevalence high risk human papilloma virus in migrant female sex workers in Spain. Sex Trans Infect. 2005; 81: 79-84.

44. Aral SO, Over M, Manhart L, et al. Sexually transmitted infections. In: Jamison DT, Breman JG, Measham AR, et al. (Eds), Disease Control Priorities in Developing Countries, 2nd ed., Washington (DC): The International Bank for Reconstruction and Development/The World Bank; New York; Oxford University Press, 2006.

45. Abdullah AS, Fielding R, Hedley AJ, et al. Risk factors for sexually transmitted diseases and casual sex among Chinese patients attending sexually transmitted disease clinics in Hong Kong. Sex Trans Dis. 2002; 29(6): 360-5.

46. Crosby RA, Diclemente RJ, Wingood G, et al. Adjudication history and African American adolescents risk for acquiring STDs: An explorer analysis. Sex Trans Dis. 2003; 30(8): 634-8.

47. Were J, Mohammed H, Furegato M, et al. Who is being diagnosed with bacterial sexually transmitted infections through partner notification? A comparison of persons diagnosed with chlamydia or gonorrhea through partner notification and those diagnosed otherwise in genitourinary medicine clinics in England, 2013. J STD AIDS. 2015; 26(11Supl) [Abstract]. 